\title{
Demokratiemodelle in den Autonomen Gemeinschaften Spaniens: Zwischen verfassungsrechtlicher Prägung und Pluralismus durch autonome Gestaltung
}

Karl Kössler

\section{Einleitung}

"Die Staatsform des spanischen Staates ist die parlamentarische Monarchie." (Art. 1 Abs. 3 der spanischen Verfassung von 1978 - spanVerf) Diese kurze, wenngleich programmatische Bestimmung scheint unmissverständlich klarzustellen, dass das postfranquistische Spanien die wiedererrungene Demokratie vorwiegend als repräsentative Demokratie versteht, in welcher die Parlamentarier als Hauptakteure fungieren. In jedem Fall war diese Entscheidung 1978 eine ganz deutliche Absage an eine semi-direkte Demokratie auf mehreren Regierungsebenen, wie sie in der Schweiz insbesondere seit der Verfassungsrevision von 1874 besteht. ${ }^{1}$ Im europäischen Vergleich steht Spanien damit eindeutig dem deutschen Modell näher, das bekanntlich ebenfalls eine Vorherrschaft der repräsentativen Demokratie und Parteiendemokratie vorsieht (Art. 20 und 21 deutsches Grundgesetz). Das berühmte Bonmot, dass direkte Demokratie eine »Prämie für jeden Demagogen $\ll^{2}$ darstelle, spiegelt schließlich nicht nur die Haltung vieler Schöpfer des deutschen Grundgesetzes wider. Ähnlich wie in Deutschland 1949, sollte die repräsentative Demokratie auch fast drei Jahrzehnte später in Spanien ein Bollwerk gegen einen Rückfall in diktatorische Zeiten bilden. Eine weitere Parallele zu Deutschland ist die starke Stellung der Parteien, die mit der Vorherrschaft der repräsentativen Demokratie natürlich in einem engen funktionalen Zusammenhang stehen. Unter dem Regime

1 Vgl. Zoltán T. Pállinger, "Direct Democracy in the Swiss Federation«, in Cristina Fraenkel-Haeberle et al. (Hrsg.), Citizen Participation in Multi-level Democracies (Brill, 2015), 253ff.

2 Theodor Heuss während der Beratungen im Parlamentarischen Rat 1948, zitiert nach Manfred G. Schmidt, Demokratietheorien: Eine Einführung (5. Aufl., Springer, 2010), 350. 
von General Franco waren die Parteien hingegen noch als Gefahr für die staatliche Einheit und für effizientes Regieren gebrandmarkt worden. ${ }^{3}$

Dieser Beitrag setzt sich zum Ziel, die Grenzen der Dominanz der repräsentativen Demokratie auf der regionalen Ebene auszuloten. Die zentrale Frage ist folglich, inwieweit das 1978 verfassungsrechtlich verankerte nationale Demokratiemodell auf die Ebene der Autonomen Gemeinschaften (AG) durchschlug und nach wie vor durchschlägt. Dies erfordert zunächst einen kurzen Abriss jener demokratierelevanten und demokratieprägenden Bestimmungen der Spanischen Verfassung, welche das nationale Modell konstituieren (Abschnitt 2). Dieses bildet dann den notwendigen Hintergrund für die darauffolgende Analyse des Demokratiemodells (oder der voneinander abweichenden Modelle) der AG, wobei insbesondere die seit 2006 reformierten Autonomiestatute zu berücksichtigen sein werden (Abschnitt 3). Die Schlussfolgerungen fassen die Ergebnisse der Analyse zusammen und stellen diese in einen vergleichenden Kontext (Abschnitt 4).

Zuvor sind allerdings noch einige grundlegende Ausführungen zu gewissen zentralen Begriffen erforderlich. Für Abweichungen der AG vom verfassungsrechtlich geprägten nationalen Demokratiemodell sind deren Autonomiestatute von entscheidender Bedeutung. »Im Rahmen der vorliegenden Verfassung « bilden diese »die grundlegende institutionelle Norm der jeweiligen Autonomen Gemeinschaft« (Art. 147 Abs. 1 spanVerf). Da die Autonomiestatute nicht nur inhaltliche Grenzen durch die Verfassung finden, sondern jedenfalls immer nach einem bilateralen Verhandlungsprozess vom spanischen Parlament per Organgesetz angenommen werden müssen (Art. 147 Abs. 3 spanVerf) wird kontrovers diskutiert, ob diese als "echte" Verfassungen gelten können. Die Qualifizierungen reichen von »secondary constitutional laws « ${ }^{4}$ und "quasi-konstitutionellen Normen $\aleph^{5}$ bis zu jenen, welche die Statute auf Grund der Rolle nationaler Institutionen

3 Vgl. Victor Ferreres Comella, The Constitution of Spain: A Contextual Analysis (Hart Publishing, 2013), 35.

4 Rosario Serra und Pablo Oñate, »The Reform of the Spanish Subnational Constitutions: Rules and Regulations and Political Contexts«, (June 2007) VII World Congress on Constitutional Law, 1, http://camlaw.rutgers.edu/statecon/workshop11greece07/workshop11/Serra.pdf (abgerufen am 03.01.2018).

5 César Colino, „Den spanischen Föderalismus vertiefen oder transformieren? Die neue Reformrunde und die Herausforderungen für den Autonomiestaat", in Europäisches Zentrum für Föderalismus-Forschung Tübingen (Hrsg.), Jabrbuch des Föderalismus 2007 (Nomos, 2008), 385. 
im Entscheidungsprozess als "not a true constitution " 6 betrachten. Da jedoch eine solche Rolle in mehreren föderalen Systemen besteht, ${ }^{7}$ scheint dies eine Bezeichnung der Statute zumindest als Quasi-Verfassungen nicht auszuschließen. Entscheidend ist für das Thema dieses Beitrags, ob und inwieweit die AG Gestaltungsspielraum im Rahmen ihrer Statutsautonomie - oder "Quasi-Verfassungsautonomie« - besitzen und diesen Spielraum auch tatsächlich nützen, um vom nationalen Demokratiemodell abzuweichen.

Weitere grundlegende Ausführungen sind zum Begriff des Demokratiemodells notwendig, wie er im Rahmen dieses Beitrags verstanden wird. Dabei geht es um die individuelle Mischung von Instrumenten repräsentativer, direkter und partizipativer Demokratie. Erstere wurde von vielen Verfassungstheoretikern unmittelbar mit dem Konzept der liberalen Demokratie verknüpft ${ }^{8}$ und stellt auch heute aus empirischer Perspektive nach wie vor die "eigentliche Form der Demokratie darstellt. ${ }^{9}$ Dennoch setzte sich im Lauf der Geschichte vielerorts die Ansicht durch, dass die repräsentative Demokratie einer Ergänzung bedarf. Dies geschah zunächst durch die Einführung der klassischen direktdemokratischen Instrumente wie Referendum und Volksinitiative und erst viel später durch Elemente der partizipativen Demokratie. Wenngleich letzterer Begriff in verschiedenen Verfassungsordnungen sowie in der theoretischen Literatur sehr unterschiedlich gebraucht wird, umfasst "partizipative Demokratie« im Rahmen dieses Beitrags sowohl deliberative Demokratie ${ }^{10}$ als auch partizipative Demokratie in einem engeren Sinn. ${ }^{11}$ Wenngleich die oftmalige

6 Juan José Solozábal, „Spain: A Federation in the Making?«, in Joachim J. Hesse und Vincent Wright (Hrsg.), Federalizing Europe? The Costs, Benefits and Preconditions of Federal Political Systems (Oxford University Press, 1996), 247.

7 Vgl. Francesco Palermo und Karl Kössler, Comparative Federalism: Constitutional Arrangements and Case Law (Hart Publishing, 2017), $265 \mathrm{ff}$.

8 Vgl. Laurence Morel, "Referendum«, in Michel Rosenfeld and Andras Sajó (Hrsg.), The Oxford Handbook of Comparative Constitutional Law (Oxford University Press, 2012), 502.

9 Ernst-Wolfgang Böckenförde, »Mittelbare/repräsentative Demokratie als eigentliche Form der Demokratie: Bemerkungen zu Begriff und Verwirklichungsproblemen der Demokratie als Staats- und Regierungsfrom", in Georg Müller et al. (Hrsg.), Staatsorganisation und Staatsfunktion im Wandel (Helbing \& Lichtenhahn, 1982) $301 \mathrm{ff}$.

10 Vgl. etwa Andreas Føllesdal, »The Value Added by Theories of Deliberative Democracy: Where (not) to Look«, in Samantha Bosson and José Luis Martì (Hrsg.), Deliberative Democracy and its Discontents (Ashgate, 2006), 57ff.

11 Vgl. etwa Benjamin Barber, Strong Democracy: Participatory Politics for a New Age (University of California Press, 1984). 
Differenzierung zwischen diesen beiden Demokratiebegriffen Unterschiede hinsichtlich des Ursprungs und der Betonung von rationaler Deliberation bzw. breiter Partizipation zum Ausdruck bringt, haben deliberative Demokratie und "partizipative Demokratie« in einem engeren Sinn doch sehr viel gemeinsam. ${ }^{12}$ Beide zielen auf eine Abschwächung der negativen Effekte des Mehrheitsprinzips ab, die auf eine Ja-oder-Nein-Entscheidung hinausläuft. Auch wenn diese letztendlich durch Parlamentarier oder in einem Referendum getroffen wird, soll die Einbeziehung in die Vorbereitung der Entscheidung und Deliberation eine breitere gesellschaftliche Akzeptanz des Ausgangs fördern.

Aus der Sicht des Föderalismus ermöglicht die zuvor erwähnte »QuasiVerfassungsautonomie« von Regionen häufig ein bis zu einem gewissen Grad abweichendes Demokratiemodell. Eine individuelle Mischung repräsentativer, direkter und partizipativer Demokratie kann dann der spezifischen politischen Kultur in einer Region entsprechen, wobei ein »democratic background consensus " auf dieser Ebene wohl auch leichter zu finden ist als auf der nationalen Ebene. ${ }^{13}$ Dass es in der Tat sehr unterschiedliche Präferenzen bezüglich des Mischverhältnisses der soeben genannten Demokratieformen geben kann, zeigen etwa die Kantone der Schweiz genauso wie die Regionen Italiens. Ob auch die spanischen AG in Abweichung vom nationalen Muster unterschiedliche Demokratiemodelle aufweisen, soll in diesem Beitrag erörtert werden.

\section{Das nationale Demokratiemodell}

\subsection{Rechte und Prinzipien: Partizipation durch repräsentative Demokratie als Regelfall}

Bezüglich des Demokratiemodells auf der nationalen Ebene ist es wichtig, zwischen Grundrechten und Prinzipien einerseits und konkreten Instrumenten andererseits zu unterscheiden. Als übergeordnetes Prinzip ist Bürgerbeteiligung, verstanden in einem sehr weiten Sinn, bereits am Anfang der Verfassung verankert: »Den öffentlichen Gewalten obliegt es ... die

12 Vgl. Karl Kössler, »Laboratories of Democratic Innovation? Direct, Participatory, and Deliberative Democracy in Canadian Provinces and Municipalities«, in Fraenkel-Haeberle et al. (Hrsg.), Citizen Participation (Fn. 1), $288 \mathrm{f}$.

13 Vgl. Karl-Peter Sommermann, „Citizen Participation in Multi-Level Democracies: An Introduction«, in Fraenkel-Haeberle et al. (Hrsg.), Citizen Participation (Fn. 1), 4. 
Teilnahme aller Bürger am politischen, wirtschaftlichen, kulturellen und sozialen Leben zu fördern. (Art. 9 Abs. 2 spanVerf) Diese allgemein gehaltene Bestimmung findet ihre Konkretisierung dann insbesondere in verschiedensten Grundrechten wie dem Recht auf Bildung (Art. 27) und der Gewerkschaftsfreiheit (Art. 28).

In Hinblick auf die grundrechtliche Dimension des nationalen Demokratiemodells stellt aber in jedem Fall Art. 23 Abs. 1 spanVerf die zentrale Bestimmung dar: »Die Bürger haben das Recht, an den öffentlichen Angelegenheiten direkt oder durch in periodischen, allgemeinen Wahlen frei gewählte Vertreter teilzunehmen."Während hier auf den ersten Blick direkte und repräsentative Demokratie gleichrangig nebeneinander stehen, so ergibt eine systematische Interpretation dieses Grundrechts doch eine sehr eindeutige Tendenz zu letzterer Form der politischen Partizipation. ${ }^{14}$ Das spanische Verfassungstribunal betonte den zweidimensionalen Aufbau des Art. 23 Abs. 1 spanVerf, indem er die von dieser Bestimmung umfassten Instrumente der politischen Beteiligung ausdrücklich den beiden Kategorien der repräsentativen Demokratie bzw. direkten Demokratie zuordnete. Unter letztere subsumierte das Höchstgericht die verschiedenen Formen eines Referendums (referéndum), ${ }^{15}$ die Volksinitiative gemäß Art. 87 Abs. 3 spanVerf (iniciativa popular), ${ }^{16}$ die offenen Räte in den Gemeinden Art. 140 spanVerf (concejo abierto). ${ }^{17}$ Im Umkehrschluss bedeutet diese Zuordnung durch das Verfassungstribunal auch, dass alle in der taxativen Aufzählung fehlenden Instrumente nicht unter Art. 23 Abs. 1 fallen, was zuweilen als eine zu restriktive Auslegung kritisiert wird. ${ }^{18}$ Dies betrifft insbesondere die in seit 2006 reformierten Autonomiestatuten verankerten Rechte auf administrative Partizipation. ${ }^{19}$

14 Für eine ausführliche Abhandlung vgl. Maria Reyes Pérez Alberdi, El derecho fundamental a participar en los asuntos públicos (Congreso de los Diputados, 2013).

15 STC 63/1987. Siehe unten 2.2.

16 STC 76/1994. Siehe unten 2.2.

17 STC 119/1995. Da sich dieser Beitrag auf die Demokratiemodelle der nationalen und regionalen Ebenen konzentriert, wird auf die lokale Ebene nicht eingegangen. Vgl. zum concejo abierto Luis Cosculluela Montaner, "El concejo abierto«, in Silvia Ballesteros Arribas et al. (Hrsg.), Administración local (La Ley, 2011), $363 \mathrm{ff}$ sowie zu Volksbefragungen in den Gemeinden Esther Martín Núñez, »The Referendum and Popular Consultations in the Autonomous State«, (2012) 4 Perspectives on Federalism 150.

18 Vgl. Josep Maria Castellà Andreu, Los derechos constitucionales de participación política en la administración pública (Cedecs, 2001), 203.

19 Siehe 3.2. 
Darüber hinaus stellte das Verfassungstribunal in derselben grundlegenden Entscheidung auch fest, dass Partizipation gemäß Art. 23 Abs. 1 »normalerweise durch gewählte Vertreter und nur ausnahmsweise direkt durch das Volk selbst ausgeübt wird $\aleph^{20}$ Diese Doktrin des außergewöhnlichen Charakters der direkten Demokratie hat sich seitdem gefestigt. In einem Urteil 2008 in Bezug auf das Baskenland bekräftigte das Verfassungstribunal abermals, dass »in unserem System der repräsentativen Demokratie ... die direkte Partizipation an öffentlichen Angelegenheiten auf jene Fälle beschränkt ist, in denen die Verfassung dies ausdrücklich vorsieht ... oder dies, ebenfalls ausdrücklich vorgesehen, durch die Vertretung des souveränen Volkes (das spanische Parlament oder eine seiner Kammern) zugelassen wird ... [Die repräsentative Demokratie] wird ergänzt durch bestimmte Instrumente direkter Demokratie, die gemäß der Verfassung die repräsentative Demokratie nicht abwerten oder ersetzen dürfen, sondern diese stützen müssen. «21

\subsection{Instrumente: Formen direkter Demokratie am Gängelband der Regierung}

Über Grundrechte und Prinzipien hinaus wird das nationale Demokratiemodell insbesondere von den konkreten Instrumenten der politischen Partizipation geprägt. Gemäß dem bereits kurz erwähnten Art. 87 Abs. 3 spanVerf regelt ein Organgesetz ${ }^{22}$ die Formen der Durchführung und die Voraussetzungen der Volksinitiative zur Einreichung von Gesetzesvorlagen. Dieses Organgesetz wurde schließlich 1984 verabschiedet ${ }^{23}$ was durch den politischen Kontext einer sich damals gerade (ver)festigenden Parteienherrschaft auf eine sehr restriktive Weise erfolgte. ${ }^{24}$ Die Präambel dieses konkretisierenden Rechtsakts begründete dies, ähnlich dem obigen Heuss-Zitat, damit, dass nach den waus der Geschichte zu ziehenden Lehren direkte Willensäußerungen des Volkes sehr leicht demagogischen Manipulationen den Weg bereiten können."Zwar wurde die Volksinitiative mit einem Organgesetz 2006 durch die Streichung einiger Ungültigkeitsgründe und die

20 STC 119/1995, FJ 3. Übersetzung und kursive Hervorhebung durch den Autor.

21 STC 103/2008, FJ 2. Siehe unten 2.2.

22 Organgesetze erfordern die Zustimmung einer absoluten Mehrheit der Mandatare im Abgeordnetenhaus (Art. 81 spanVerf).

23 Organgesetz Nr. 3 vom 26. März 1984.

24 Vgl. Nicolás Pérez-Sola, »Algunas propuestas para la reforma de la Ley Orgánica de Iniciativa Legislativa Popular«, (1994) 84 Revista de la Facultad de Derecho de la Universidad Complutense 481. 
Erleichterung der Unterschriftensammlung (z.B. längerer Zeitraum und elektronische Unterschriften) reformiert, ${ }^{25}$ dennoch bildet die geltende rechtliche Regelung aber nach wie vor ein sehr enges Korsett.

Bereits auf Grund von Art. 87 Abs. 3 spanVerf ist jedenfalls eine Mindestzahl von 500000 beglaubigten Unterschriften notwendig. Im Vergleich zur berühmten Volksinitiative auf Total- bzw. Teilrevision der Bundesverfassung in der Schweiz (Art. 138 und 139 der schweizerischen Verfassung), die 100000 Unterschriften erfordert, scheint die Mindestzahl im Fall Spaniens proportional zur jeweiligen Gesamtbevölkerung nicht exorbitant hoch zu sein. Dabei ist jedoch auch zu beachten, dass Art. 87 Abs. 3 spanVerf im Gegensatz zu den genannten schweizerischen Beispielen nicht eine Verfassungsinitiative, sondern eine einfache Gesetzesinitiative betrifft. In Bezug auf Gesetzesinitiativen sieht etwa der vergleichbare Art. 71 Abs. 2 der italienischen Verfassung vor, dass die Unterschriften von lediglich 50000 Wählern genügen.

Dennoch liegt die Krux der spanischen Volksinitiative in anderen, weit gravierenderen Schwachstellen. Art. 87 Abs. 3 spanVerf legt ausdrücklich fest, dass eine Volksinitiative in Steuersachen, internationalen Fragen sowie bezüglich des Begnadigungsrechts unzulässig ist, womit die Einschränkungen des aufhebenden Referendums gemäß Art.75 der italienischen Verfassung beinahe wortgleich übernommen wurden. ${ }^{26}$ Außerdem ist eine Volksinitiative auch bezüglich weiterer organgesetzlich zu regelnder Materien unzulässig. Insbesondere der letzte Ausschlussgrund ist schwerwiegend und von großer Tragweite, da die spanische Verfassung Organgesetzen sehr breiten Raum gibt und diese viele Inhalte von besonderer Bedeutung betreffen. ${ }^{27}$ Auch wenn sich dies nicht explizit in den Ausschlussgründen des Art. 87 Abs. 3 spanVerf findet, so ist durch die Konzeption als Gesetzesinitiative auch die Anregung von Verfassungsänderungen nicht möglich. Volksinitiativen mit so weitreichenden Folgen wie etwa jene, die 2002 in der Schweiz zur Entscheidung über den Beitritt zu den Vereinten Nationen führte, wären also jedenfalls unzulässig.

25 Organgesetz Nr. 4 vom 26. Mai 2006.

26 Vgl. Víctor Cuesta-López, »The Spanish Agenda Initiative and the Reform of its Legal Regime: A New Chance for Participatory Democracy?«, in Maija Setälä und Theo Schiller (Hrsg.), Citizens' Initiatives in Europe: Procedures and Consequences of Agenda-Setting by Citizens (Palgrave Macmillan, 2012), 198.

27 Wichtige Organgesetze sind etwa folgende: LOFCA (Ley Orgánica de Financiación de las Comunidades Autónomas), LOAPA (Ley Orgánica de Armonización del Proceso Autonómico), LOREG (Ley Orgánica de Régimen Electoral General) und nicht zuletzt auch die Autonomiestatute. 
Eine weitere starke Einschränkung der Wirksamkeit der Volksinitiative bildet die Möglichkeit einer Mehrheit im Abgeordnetenhaus (Congreso de los Diputados), die Gesetzesvorlage nicht zu berücksichtigen. In der Tat lehnte die erste Kammer des spanischen Parlaments bisher die Beratung fast aller Volksinitiativen ab. Eine Ausnahme bildete lediglich eine Vorlage zur Regelung der Kostentragung innerhalb von Wohnungseigentümergemeinschaften. Selbst in diesem Ausnahmefall ging die Initiative aber nur teilweise vom Volk aus, zumal zwei Parlamentsfraktionen ebenfalls Gesetzesentwürfe zum Thema eingebracht hatten und diese drei Vorlagen gemeinsam debattiert wurden. ${ }^{28}$

Neben der Volksinitiative trifft die spanische Verfassung mit verschiedensten Formen von Referenden eine weitere Richtungsentscheidung für das Demokratiemodell auf der nationalen Ebene. Dabei muss zwischen rechtlich unverbindlichen und verbindlichen Volksabstimmungen unterschieden werden. Ein Votum der letzteren Art kann insbesondere im Zuge von Verfassungsänderungen abgehalten werden, die im Normalfall DreiFünftel-Mehrheiten in beiden Kammern des spanischen Parlaments (Art. 167 Abs. 1 spanVerf) oder eine Drei-Viertel-Mehrheit im Abgeordnetenhaus bei gleichzeitiger absoluter Mehrheit im Senat erfordern (Art. 167 Abs. 2 spanVerf). Innerhalb von 15 Tagen nach der Annahme kann ein Zehntel der Mitglieder einer der beiden Kammern die Durchführung einer Volksabstimmung verlangen (Art. 167 Abs. 3 spanVerf). Bei einer Gesamtrevision oder Änderung von Bestimmungen gewisser besonders geschützter Titel der Verfassung muss sogar eine Drei-Viertel-Mehrheit beider Kammern vor ihrer Auflösung sowie nach ihrer sofortigen Neuwahl gegeben sein (Art. 168 Abs. 1-2 spanVerf). Überdies ist in diesem Fall eine Volksabstimmung obligatorisch und nicht nur auf Antrag von Parlamentariern abzuhalten (Art. 168 Abs. 3 spanVerf). Auch eine Lösung der gegenwärtigen Katalonienkrise mittels einer Verfassungsänderung, was von der sozialdemokratischen PSOE (Partido Socialista Obrero Español) zuweilen vorgeschlagen wurde, müsste nach der letztgenannten erschwerten Prozedur erfolgen. Neben dem obligatorischen Referendum stellt diesbezüglich auch die Drei-Viertel-Mehrheit im spanischen Parlament eine praktisch unüberwindbare Hürde dar, umso mehr, da die dünner besiedelten Zentralregionen Spaniens nach dem geltenden Wahlrecht im Abgeordnetenhaus und noch stärker im Senat im Vergleich zu Katalonien und dessen 
potenziellen Bündnispartnern wie das Baskenland überrepräsentiert sind. ${ }^{29}$

Ein inhaltlich weitreichenderes Instrument als die Verfassungsreferenden gemäß Art. 167-168 spanVerf ist in Art. 92 spanVerf verankert. Diese Bestimmung erlaubt es dem spanischen Ministerpräsidenten nach Genehmigung durch eine absolute Mehrheit des Abgeordnetenhauses, "politische Entscheidungen von besonderer Tragweite« einer beratenden Volksabstimmung zu unterwerfen. Wie schon im Fall der Volksinitiative wird bezüglich der näheren normativen Ausgestaltung wiederum auf ein Organgesetz verwiesen.

Drei Punkte scheinen an dieser Stelle besonders bemerkenswert. Erstens hat das Referendum gemäß dem eindeutigen Wortlaut der Verfassungsbestimmung lediglich konsultativen Charakter. Demgegenüber ergibt ein europaweiter Vergleich, dass Referenden mit einer rechtsverbindlichen Entscheidung klar überwiegen und eine Reihe von Staaten überhaupt nur derartige Formen von Abstimmungen kennt. ${ }^{30}$ Zweitens ist Art. 92 spanVerf so konstruiert, dass das Referendum einen top-down-Charakter hat. Die Initiative geht schließlich nicht vom Volk aus, sondern vom Ministerpräsidenten, der im Regelfall auch über die geforderte absolute Mehrheit im Abgeordnetenhaus verfügt. Dies kontrastiert wiederum insbesondere mit den vielen Volksabstimmungen in der Schweiz auf der nationalen, kantonalen und kommunalen Ebene, die gemäß Art. 138 und 139 der Schweizerischen Verfassung bzw. den jeweiligen Kantonsverfassungen auf Volksinitiativen zurückgehen. Dass das Referendum im spanischen Fall eine Regierungsinitiative darstellt, wurde durch die konkretisierende organgesetzliche Regelung 1980 noch zusätzlich verstärkt. ${ }^{31}$ Jener Rechtsakt rückt nämlich bezüglich der konkreten Verfahrensregeln die Regierungspartei(en) noch mehr ins Zentrum, sodass die Volksabstimmung gemäß Art. 92 spanVerf einer parteipolitischen »Rationalisierung « ${ }^{32}$ unterzogen wurde. Eine dritte Schwachstelle des Referendums betrifft die Einschrän-

29 Vgl. Andrés Boix Palop, »The Catalunya Conundrum, Part 2: A Full-Blown Constitutional Crisis for Spain« (Verfassungsblog, 27. September 2017) https://verfassun gsblog.de/the-catalunya-conundrum-part-2-a-full-blown-constitutional-crisis-for-sp ain/ (abgerufen am 11. April 2018).

30 Vgl. Europäische Kommission für Demokratie durch Recht, Referendums in Europe: An Analysis of the Legal rules in European States (CDL-AD (2005)034), para 116-122. Die Studie unterscheidet zwischen rechtlich verbindlichen »decision-making referendums" und unverbindlichen »consultative referendums".

31 Organgesetz Nr. 2 vom 18. Januar 1980.

32 Vgl. Pedro Cruz Villalón, »El referendum consultivo como modelo de racionalización constitucional«, in Pedro Cruz Villalón, La curiosidad del jurista persa, y 
kung, dass es sich, wiederum im Gegensatz etwa zur Schweiz, in keinem Fall auf konkrete Gesetze, sondern nur auf allgemeine politische Entscheidungen beziehen darf, die von besonderer Tragweite sein müssen. Die Beurteilung der Frage, was von besonderer Tragweite ist, obliegt natürlich der Regierung.

Vor diesem Hintergrund ist es nicht verwunderlich, dass Volksabstimmungen gemäß Art. 92 spanVerf extrem selten sind. Bisher erfolgte ein derartiges Votum lediglich zwei Mal, nämlich 1986 bezüglich der NATOMitgliedschaft Spaniens sowie 2005 hinsichtlich der Ratifizierung des Vertrags über eine Verfassung für Europa (VVE). Während die Befürworter in beiden Abstimmungen die Oberhand behielten, lassen sich aus beiden Fällen wichtige Lehren in Bezug auf die zu Grunde liegenden politischen Dynamiken der Initiierung und Durchführung ziehen, die dem Ziel einer direktdemokratischen Sachentscheidung teilweise zuwiderliefen.

Beim Referendum zum VVE lässt sich die Regierungsinitiative auf externe Faktoren zurückführen. ${ }^{33}$ Andere Mitgliedstaaten hatten zuvor ebenfalls Volksabstimmungen angekündigt, welche durch die bekannten Resultate in Frankreich und den Niederlanden letztendlich das Schicksal des Vertrags besiegelten, sodass auch Spanien diesen Weg einschlug. Neben der breiten Befürwortung durch die Regierung der PSOE und die PP (Partido Popular) als Opposition, unterstützte mit 76,3 Prozent auch eine große Mehrheit der Bevölkerung den Vertrag.

Im Fall der NATO-Mitgliedschaft hatte die sozialdemokratische PSOE als Oppositionspartei den Beitritt 1982 abgelehnt und eine Abstimmung über einen Austritt versprochen, später aber (teilweise) einen Richtungswechsel zu einer pro-NATO-Haltung vollzogen. Das Referendum 1986 wurde von Ministerpräsident Felipe Gonzàlez daher auch deshalb einberufen, um innerparteilichen Widerstand in seiner PSOE durch eine direkte Berufung auf den Volkswillen zu brechen. ${ }^{34}$ Neben dieser Art von (partei)politischer Instrumentalisierung ist eine Strategie der Personalisierung eine zweite wichtige Lehre aus ebendieser Volksabstimmung. Gonzàlez hatte seine persönliche Popularität genutzt und die NATO-Entscheidung quasi in eine Vertrauensfrage hinsichtlich seiner Regierung umgedeutet. Die konkrete Sachentscheidung und der ihr vorangehende Deliberationsprozess wurde also massiv von persönlichen Motiven überlagert und beein-

otros estudios sobre la Constitución (Centro de Estudios Políticos y Constitucionales, 1999), 255.

33 Vgl. Comella, Constitution of Spain (Fn. 3), $35 \mathrm{f}$.

34 Vgl. Lawrence LeDuc, »Referendums and Deliberative Democracy« (International Political Science Association World Congress, Fukuoka, July 2006). 
trächtigt. Während sich dieses politische Kalkül beim NATO-Referendum auszahlte und entgegen der Prognosen letztlich 56,9 Prozent für die Mitgliedschaft stimmten, war eine ähnliche Personalisierung des italienischen Verfassungsreferendums 2016 durch Ministerpräsident Matteo Renzi bekanntlich nicht erfolgreich. Ein weiterer neuralgischer Punkt des NATOReferendums, der auch in einem internationalen Vergleich der Referendumspraxis sehr kritisch beurteilt wurde, betraf den Wortlaut der Fragestellung. ${ }^{35}$ Zumal das Wahlvolk über eine Mitgliedschaft "zu den von der Regierung festgelegten Bedingungen« abstimmen sollte, wurde die Debatte mehr auf nicht näher konkretisierte Bedingungen als die Mitgliedschaft selbst gelenkt und der Eindruck erweckt, dass Spanien diese Bedingungen unilateral festlegen könne. Diese politische Instrumentalisierung des unverbindlichen Referendums gemäß Art. 92 spanVerf scheint neben dem sehr engen rechtlichen Korsett dazu beigetragen zu haben, dass sich keine Referendumskultur entwickelte. Auch deshalb ist und bleibt das spanische Demokratiemodell auf der nationalen Ebene eindeutig von einer Vorherrschaft der repräsentativen Demokratie geprägt.

\section{Demokratiemodelle in den Autonomen Gemeinschaften}

\subsection{Verfassungsrechtliche Vorgaben als Grenzen regionaler Demokratiemodelle}

Was das Demokratiemodell, oder besser, die Demokratiemodelle in den AG betrifft, so ist vorauszuschicken, dass diese von zweierlei Einflüssen geprägt sind. Zum einen gibt es in einigen wenigen Fällen ganz konkrete verfassungsrechtliche Vorgaben. Zum anderen haben aber die Statute der AG auch Spielraum zur individuellen Gestaltung, der wiederum durch die verfassungsrechtlichen Vorgaben begrenzt wird.

1978 legte die Verfassung insbesondere fest, dass eine regionale Volksabstimmung jedenfalls bezüglich der Verabschiedung und der späteren Änderung der Statute gewisser AG abgehalten werden müsse (Art. 151 Abs. 1 und 152 Abs. 2 spanVerf). Dies betraf Katalonien, das Baskenland, Galizien und Andalusien, deren Statute zwar zu dieser zusätzlichen direktdemokratischen Legitimation verpflichtet wurden, aber dafür früher als jene ande-

35 Vgl. Stephen Tierney, Constitutional Referendums: A Theoretical Enquiry (Oxford University Press), 229. 
rer AG mehr Zuständigkeiten übernehmen durften. ${ }^{36}$ Dass Referenden in Bezug auf Statutsänderungen in der Praxis eine viel größere Bedeutung erlangten als die für Verfassungsreformen vorgesehenen Volksabstimmungen, zeigte sich insbesondere im Jahr 2006. Damals sprachen sich 73,9 Prozent der Wähler für das neue katalanische Autonomiestatut aus, welches dann aber bekanntlich vom Verfassungstribunal teilweise als verfassungswidrig aufgehoben bzw. verfassungskonform interpretiert wurde. ${ }^{37}$ Der Gegensatz zwischen dieser Entscheidung des Wahlvolks und der Entscheidung einer höchstrichterlichen Instanz entwickelte sich daraufhin zu einer der Ursachen der gegenwärtigen Katalonienkrise.

Ein weiterer Verfassungsartikel ist von sehr großer aktueller Bedeutung. Gemäß Art. 149 Art. 1 Z. 32 spanVerf fällt nämlich die »Genehmigung der Einberufung einer Volksbefragung auf dem Wege des Referendums « in die ausschließliche Zuständigkeit des Staates, d.h. der Zentralregierung. Da diese Bestimmung auch auf Referenden der AG anwendbar ist, lässt sich an dieser Stelle der Bogen zu den Statuten als neben der Verfassung zweiten Bestimmungsfaktor der regionalen Demokratiemodelle schlagen.

\subsection{Rechte und Prinzipien: Die reformierten Autonomiestatute und die Entdeckung partizipativer Demokratie}

Bemerkenswert ist diesbezüglich der starke Kontrast zwischen den 1979 bis 1983 verabschiedeten ursprünglichen Statuten einerseits ${ }^{38}$ und den ab 2006 reformierten Dokumenten andererseits. Erstere übernahmen weitgehend das kurz zuvor 1978 entworfene (vorwiegend repräsentative) nationale Demokratiemodell. Neben der Etablierung der repräsentativen Institutionen der AG und der Festlegung ihrer Kompetenzen, beides obligatorische Statutsinhalte gemäß Art. 147 Abs. 2 bzw. 152 Abs. 1 spanVerf, folgten die Statute weitgehend dem Muster der nationalen Verfassung. Viele

36 Zur Konstituierung der AG, vgl. Karl Kössler, Multinationaler Föderalismus in Theorie und Praxis: Kompetenz- und finanzverfassungsrechtliche Aspekte der territorialen Self-Rule in Kanada, Belgien und Spanien (Nomos, 2012), $165 \mathrm{ff}$.

37 STC 31/2010. Für eine umfassende Analyse vgl. insbesondere die Sonderausgabe »Especial Sentència 31/2010 del Tribunal Constitucional, sobre l'Estatut d' autonomia de Catalunya de 2006« der Revista catalana de dret public (2010).

38 Vgl. Eduardo J. Ruiz Vieytez, »Federalism, Subnational Constitutional Arrangements, and the Protection of Minorities in Spain«, in G. Alan Tarr, Robert F. Williams und Joseph Marko (Hrsg.), Federalism, Subnational Constitutions and Minority Rights (Praeger, 2004), 142. 
von ihnen verankerten vergleichbar dem schon erwähnten Art.9 Abs. 2 spanVerf Bürgerbeteiligung als sehr allgemeines Prinzip für die öffentlichen Gewalten, ${ }^{39}$ regelten in ähnlicher Weise das regionale Wahlrecht und gaben direktdemokratischen Instrumenten nur wenig Raum..$^{40}$

Im Gegensatz dazu sprechen die reformierten Statute die Themen Partizipation und Demokratie deutlich expliziter und umfassender an. Dies ist wohl einerseits auf einen globalen Wandel des politischen Zeitgeists zurückzuführen, andererseits aber auch in einer tiefer liegenden Unzufriedenheit mit dem bestehenden Demokratiemodell begründet. Es überrascht daher kaum, dass die neuen Statute, vor allem jene Kataloniens und der Balearischen Inseln, über die vier höchsten Werte der Rechtsordnung gemäß des Art. 1 Abs. 1 spanVerf hinaus (Freiheit, Gerechtigkeit, Gleichheit und politischer Pluralismus) als Werte Demokratie und Partizipation nennen.

Darüber hinaus verankern fast alle reformierten Statute der AG ein Recht auf Partizipation. Abgesehen davon, dass sie sich dabei auf den zuvor erwähnten Art. 23 spanVerf berufen,${ }^{41}$ sind diese Regelungen im Sinne der autonomen Gestaltungsfreiheit sehr unterschiedlich und folgen drei grundsätzlich verschiedenen Ansätzen. ${ }^{42}$ Neu war im Vergleich zu den alten Statuten insbesondere, dass man nun bezüglich der Ergänzung der repräsentativen Demokratie einem dualen Ansatz folgt und unter Partizipation neben den klassischen direktdemokratischen Instrumenten der Volksinitiative und des Referendums auch partizipative Demokratie im in der Einleitung dargelegten Sinn subsumierte.

\subsection{Instrumente: Der statutarische Dualismus von Formen direkter und partizipativer Demokratie}

In Bezug auf die neuen Instrumente partizipativer Demokratie, welche bis zu diesem Zeitpunkt in Spanien keinerlei Tradition aufgewiesen hatte, sind etwa verschiedene Formen der konsultativen Mitwirkung am Gesetzgebungsprozess in den AG zu nennen. Solche waren zuvor bestenfalls in einigen parlamentarischen Geschäftsordnungen vorgesehen und wurden

39 Siehe oben 2.1 .

40 Für einen Überblick zu den relevanten Bestimmungen in den alten Statuten, vgl. Maria Reyes Pérez Alberdi, »Democracia representativa y participación ciudadana en España« (2014) 10 federalismi.it 1, 11.

41 Siehe oben 1.

42 Vgl. Reyes Pérez Alberdi, »Democracia representativa«(Fn. 40), $11 \mathrm{f}$. 
nun auf die Statutsebene gehoben. ${ }^{43}$ Noch mehr Raum wird jedoch Instrumenten der administrativen Partizipation eingeräumt, die in Bezug auf gewisse Verwaltungsentscheidungen Informations- und Teilhaberechte garantieren. ${ }^{44}$ Zwar sehen einzelne AG wie Aragon, deren Statut dem Thema Bürgerbeteiligung besonders viel Raum gibt, ${ }^{45}$ ein allgemeines Recht auf administrative Partizipation vor (Art. 15 Abs. 2 des Statuts von Aragon). Im Normalfall ist diese Form von Beteiligung aber im Rahmen des in vielen Statuten verankerten Rechts auf gute und effiziente Verwaltung geregelt. ${ }^{46}$ Diese legistische Vorgangsweise bringt klarer das Verständnis des Verfassungstribunals zum Ausdruck, welches grundlegend zwischen politischer und administrativer Partizipation unterscheidet.

Diese Differenzierung ist von fundamentaler Bedeutung, weil die ständige Judikatur Rechte vor der Verwaltung, wie bereits angedeutet, nicht unter Art. 23 spanVerf subsumiert ${ }^{47}$ und diesen seit einer bedeutenden Entscheidung zum Statut Valencias 2007 eine Anerkennung als unmittelbar anwendbare subjektive Rechte verweigert. ${ }^{48}$ Administrative Beteiligungsrechte werden, wie etwa auch soziale Rechte, lediglich als Leitprinzipien (principios rectores) betrachtet und erfordern zur Erlangung des Status von subjektiven Rechten noch ausführende Akte der einfachen Gesetzgebung durch die AG. Andere statutarische Partizipationsrechte als jene im Verwaltungsbereich sind hingegen gemäß diesem Urteil sehr wohl als subjektive Rechte zu verstehen, da sie mit dem obligatorischen Statutsinhalt der institutionellen Organisation der Regionen (Art. 147 Abs. 2 lit.c und 152 Abs. 1 spanVerf) in einem direkten Zusammenhang stehen. Die-

43 Vgl. María Jesús Larios Paterna, La participación ciudadana en la elaboración de la ley (Congreso de los Diputados, 2003), 267ff.

44 Vgl. Enriqueta Expósito Gómez und Josep Maria Castellà Andreu, «Los derechos políticos y ante la Administración en el Estatuto de Autonomía de Cataluña«, in Josep Maria Castellà und Enriqueta Expósito Gómez (Hrsg.), Derechos y principios rectores en los Estatutos de Autonomía (Atelier, 2009), 85ff.

45 Das Statut von Aragón garantiert recht detailliert Teilhaberechte unterschiedlichster Kategorien von Individuen (Senioren, Immigranten, etc.) und Vereinigungen (Konsumentenschutzvereine, etc.). Vgl. Sergio Castel Gayan, "Civil participation policy and democratic innovation in the autonomous community of Aragon", (2012) 4 Perspectives on Federalism 230, 234.

46 Vgl. Josep Maria Castellà Andreu, „Estatutos reformados y participación ciudadana: De la ampliación de contenidos a las restricciones del Tribunal Constitucional«, in Yolanda Gómez Sánchez et al. (Hrsg.), Constitución y democracia: ayer y boy (UNED, 2012), 3617.

47 Siehe oben 2.1.

48 STC 247/2007, FJ 15. Für eine Analyse dieser Entscheidung, vgl. Palermo und Kössler, Comparative Federalism (Fn. 7), 341. 
ser Unterscheidung folgte 2010 auch das berühmte Urteil zum katalanischen Statut, ${ }^{49}$ ohne dabei jedoch ausdrücklich und klar festzulegen, welche der verankerten Rechte subjektive Rechte und welche bloß Leitprinzipen darstellen.

Wie eingangs erwähnt, beinhalten die reformierten Autonomiestatute als Ergänzung zur repräsentativen Demokratie ein duales System mit Instrumenten sowohl der partizipativen als auch der direkten Demokratie. Letztere hatten als traditionelle Instrumente bereits in den ursprünglichen Fassungen Berücksichtigung gefunden hatten. Die Frage ist auch diesbezüglich, inwieweit die Regelungen in den Statuten und ausführenden Gesetzen zu regionalen Volksinitiativen und Referenden Ausdruck eines autonomen Gestaltungswillens sind oder dem nationalen Muster folgen. Hinsichtlich der Volksinitiative ist zunächst noch einmal darauf hinzuweisen, dass das 1984 auf der Basis von Art. 87 Abs. 3 spanVerf verabschiedete Organgesetz die Materie in einer ziemlich restriktiven Art regelte. ${ }^{50} \mathrm{Im}$ Allgemeinen passte sich die Gestaltung dieses Instruments in den AG den organgesetzlichen Vorgaben an, was wohl über das Gebot der institutionellen Homogenität (Art. 152 Abs. 1 spanVerf) hinausgeht und als »clear symptom of legislative apathy ${ }^{51}$ kritisiert wurde.

Abweichungen, wenn auch relativ geringfügige, betreffen aber zum Beispiel die Regelungen bezüglich der Unterschriftensammlung. Während Art. 1 des Organgesetzes von 1984 eine Einschränkung auf mindestens 18jährige spanische Bürger festschreibt, sind gemäß Art. 2 eines katalanischen Gesetzes von $2006^{52}$ auch dauerhaft ansässige Ausländer sowie Jugendliche mit einem Mindestalter von 16 Jahren zur Unterschrift berechtigt. Die geforderte Mindestanzahl an Unterschriften für eine regionale Volksinitiative variiert recht stark zwischen 0,7 Prozent des Elektorats in Galizien und 5 Prozent in Extremadura, wobei das bekanntlich verfassungsrechtlich festgelegte Minimum von 500000 für nationale Initiativen $^{53}$ einem Anteil von 1,5 Prozent entspricht. ${ }^{54}$ Was die thematischen Einschränkungen betrifft, so sehen die regionalen Bestimmungen, ähnlich den verfassungsrechtlichen und organgesetzlichen Vorgaben für die nationale Ebene, bestimmte ausgeschlossene Inhalte vor. Typischerweise betreffen die Ausschlussgründe Gesetzesinitiativen in Bezug auf die Institutio-

49 STC 31/2010.

50 Organgesetz Nr. 3 vom 26. März 1984. Siehe oben 2.2.

51 Cuesta-López, »Spanish Agenda Initiative« (Fn. 26), 195.

52 Gesetz Nr. 1 vom 16. Februar 2006.

53 Siehe oben 2.2.

54 Vgl. Cuesta-López, »Spanish Agenda Initiative«(Fn. 26), 197. 
nen, das Wahlsystem, den Gesetzgebungsprozess in den AG sowie die Volksinitiative selbst. ${ }^{55}$ Katalonien lässt diesbezüglich abermals etwas mehr Partizipation zu, indem 300000 Bürger im Gegensatz zu allen anderen Regionen eine Reform des Autonomiestatuts initiieren können (Art. 222 Abs. 1a und Art. 223 Abs. 1a des katalanischen Statuts).

Lenkt man den Blick von der rechtlichen Regelung der Volksinitiative in den AG auf deren Umsetzung in der Praxis, so treten einige regionale Besonderheiten zu Tage. Der größte Teil der Volksinitiativen entfällt eindeutig auf die Kanarischen Inseln, wobei sich mehrere Erklärungsmuster anbieten. ${ }^{56}$ Abgesehen von einer eventuell besonders aktiven demokratischen Kultur in dieser Autonomen Gemeinschaft fällt auf, dass dort die Hemmschwelle für die Unterschriftensammlung mit 1 Prozent der Wahlbevölkerung besonders niedrig angesetzt ist. Ein weiterer Faktor scheint der positive Anreiz einer relativ hohen Erfolgsrate zu sein, zumal auf den Kanarischen Inseln auch vergleichsweise viele Volksinitiativen zur parlamentarischen Beratung angenommen wurden. Dies steht in starkem Kontrast zur, wie schon erwähnt, lediglich einmaligen parlamentarischen Debatte einer solchen Initiative auf der nationalen Ebene. ${ }^{57}$ In der Tat scheint ganz allgemein die hohe Ablehnungsrate durch die jeweilige gesetzgebende Versammlung ein wichtiger Grund dafür zu sein, dass die Volksinitiative national und in vielen AG ein relativ zahnloses Instrument bleibt. ${ }^{58}$ Andere Faktoren wie der Ausschluss einer Vielzahl an Materien sowie die Nichterreichung der Mindestanzahl an Unterschriften könnten hingegen durch Änderungen der rechtlichen Rahmenbedingungen sehr wohl beeinflusst werden. Nur machten die AG von ihrem Gestaltungsspielraum bisher kaum Gebrauch.

Neben der Volksinitiative stellen Formen von Volksbefragungen (consultas populares) das zweite erwähnenswerte Instrument der direkten Demokratie dar, dem in den reformierten Statuten auch vergleichsweise mehr Aufmerksamkeit gewidmet wird. Aus rechtstechnischer Perspektive wird vereinzelt ein (vages) Recht auf Einforderung einer Volksbefragung durch die Regionalregierung verbrieft (z.B. Art. 29 Abs. 6 des Statuts von

55 Vgl. Vicente José Cabedo-Mallol, »La iniciativa legislativa popular en las Comunidades Autónomas: La necesaria reforma de su legislación«, (2009) 24 Teoría y Realidad Constitucional 455, 460.

56 Vgl. Comella, Constitution of Spain (Fn. 3), 36f; Cuesta-López, »Spanish Agenda Initiative« (Fn. 26), 204 und 208f.

57 Siehe oben 2.2 .

58 Vgl. Victor Cuesta-López, Participación directa e iniciativa legislativa del ciudadano en democracia constitucional (Thomson-Civitas, 2008), $417 \mathrm{f}$. 
Katalonien), in den meisten Fällen aber eine Kompetenz der Autonomen Gemeinschaft bezüglich eines solchen Votums festgeschrieben. Einige Autonomiestatute verankern dabei eine exklusive regionale Kompetenz (z.B. Art. 122 des Statuts von Katalonien). Andere wiederum sehen eine mit dem Staat geteilte Zuständigkeit vor (z.B. Art. 50 Abs. 8 des Statuts von Valencia). ${ }^{59}$ Für die meisten Kontroversen sorgte und sorgt jedoch die Frage, ob die so oft erwähnten vielgestaltigen Formen der Volksbefragung auch Referenden miteinschließen. Mehrere AG stellen ausdrücklich klar, dass letztere nicht umfasst sind (z.B. Art. 71 Abs. 27 des Statuts von Aragon). Art. 122 des katalanischen Statuts verankert allerdings recht kryptisch eine regionale Zuständigkeit in Bezug auf »Umfragen, öffentliche Anhörungen, Foren der Partizipation und jegliches anderes Instrument der Volksbefragung mit Ausnahme der Bestimmungen des Art. 149 Abs. 1 Z. 32 spanVerf." Entscheidend ist folglich die Interpretation dieses bereits angesprochenen Verfassungsartikels demzufolge die »Genehmigung der Einberufung einer Volksbefragung auf dem Wege des Referendums « eine ausschließliche Kompetenz der Zentralregierung darstellt.

$\mathrm{Zu}$ ebendiesem Thema nahm das Verfassungstribunal 2008 in Bezug auf das Baskenland Stellung. Nachdem der Entwurf für ein reformiertes Statut (»Plan Ibarretxe«), das eine »libre asociación « zwischen dem Baskenland und Spanien vorgesehen hatte, 2005 im Abgeordnetenhaus wegen Bedenken bezüglich der Verfassungsmäßigkeit dieses Modells gescheitert war, wurde für 2008 eine nicht-verbindliche Volksbefragung (consulta popular) anberaumt. Zur Abstimmung kam dabei die Frage, ob »die baskischen Parteien, ohne Ausnahme, einen Verhandlungsprozess initiieren sollen, um eine demokratische Vereinbarung über das Recht des baskischen Volkes zur Entscheidung über seinen politischen Status zu erreichen, und diese Vereinbarung vor 2010 einem Referendum unterworfen werden soll." Das Verfassungstribunal stellte jedoch fest, dass Art. 149 Abd. 1 Z. 32 spanVerf auch für eine rechtlich nicht verbindliche Abstimmung die Genehmigung der Einberufung durch die Zentralregierung voraussetze und darüber hinaus die gesamte Regelung des Rechtsinstituts des Referendums in die nationale Zuständigkeit falle. ${ }^{60}$ In seiner berühmten Entscheidung zum katalanischen Statut berief sich das Verfassungstribunal zwei Jahre später auf

59 Vgl. Josep Maria Castellà Andreu, »Principles, Rights and Participatory Institutions in the Reformed Statutes", (2012) 4 Perspectives on Federalism 20, $30 \mathrm{ff}$.

60 STC 103/2008. Für eine ausführliche Analyse dieses Urteils, vgl. Alberto LópezBasaguren, "Sobre referéndum y Comunidades Autónomas. La ley vasca de la >consulta< ante el Tribunal Constitucional«, (2009) 9 Revista d'Estudis Autonòmics $i$ Federals 202, $202 \mathrm{ff}$. 
diese Doktrin und schränkte somit den zuvor angesprochenen Art. 122 des Statuts von Katalonien massiv ein. ${ }^{61}$ Das Thema Volksbefragung beherrscht natürlich auch die gegenwärtige Katalonienkrise. Nachdem das spanische Parlament die Genehmigung eines Referendums abgelehnt hatte, hielt die katalanische Regierung am 9. November 2014 unilateral und entgegen einer einstweiligen Verfügung des Verfassungstribunals eine Volksbefragung zur Unabhängigkeit ab, welche daraufhin für verfassungswidrig erklärt wurde. ${ }^{62}$ Ein ähnliches Wechselspiel zwischen dem Höchstgericht und der katalanischen Regierung fand bekanntlich in den Folgejahren bis zum Unabhängigkeitsvotum am 1. Oktober 2017 statt. $^{63}$

Abseits der soeben erörterten Kontroverse rund um Volksbefragungen der AG, rechtlich gesprochen also um das Verhältnis zwischen Art. 149 Abd. 1 Z. 32 spanVerf und den jeweiligen Statutsbestimmungen, etablierten die reformierten Autonomiestatute in einem spezifischen Bereich sehr wohl verbindliche regionale Referenden. An dieser Stelle muss noch einmal in Erinnerung gerufen werden, dass für Statutsänderungen in Katalonien, dem Baskenland, Galizien und Andalusien seit jeher eine Volksabstimmung direkt auf Grund der Verfassung vorgesehen war (Art. 151 Abs. 1 und 152 Abs. 2 spanVerf). Mit ihren 2006-2011 reformierten Statuten führten Valencia, Aragon und Extremadura dieses Votum zu Änderungen ihrer Quasi-Verfassungen selbst ein, wenngleich nicht als obligatorisch. In Valencia kann die Abstimmung etwa im Fall einer bloßen Kompetenzerweiterung entfallen (Art. 81 Abs. 5 des Statuts), während sie in den beiden anderen AG nur auf Antrag einer Zwei-Drittel-Mehrheit des Regionalparlaments abgehalten wird (Art. 115 Abs. 7 des Statuts von Ara-

61 STC 31/2010, FJ 69. Für nähere Details, vgl. Josep Maria Castellà Andreu, «Las consultas populares en la Sentencia 31/2010, sobre el Estatuto de Autonomía de Cataluña«, in Enrique Álvarez Conde und Cecilia Rosado Villaverde (Hrsg.), Estudios sobre la Sentencia 31/2010, de 28 de junio, del Tribunal Constitucional sobre el Estatuto de Autonomía de Cataluña (IDP, 2011), $197 \mathrm{ff}$.

62 STC 31/2015 und STC 32/2015.

63 Für einen Überblick über die Ereignisse bis 2017, vgl. Andrés Boix Palop, »The Catalunya Conundrum, Part 1: How Could Things Come to Such a Pass? « (Verfassungsblog, 26. September 2017) https://verfassungsblog.de/the-catalunya-conundru m-part-1-how-could-things-come-to-such-a-pass/ (abgerufen am 11. April 2018); Victor Ferreres Comella, »The Catalan Secessionist Movement and Europe - Remarks on the Venice Commission's Opinion 827/2015« (Verfassungsblog, 22. März 2017) https://verfassungsblog.de/the-catalan-secessionist-movement-and-europe-re marks-on-the-venice-commissions-opinion-8272015/ (abgerufen am 11. April 2018). 
gon bzw. Art. 91 Abs. 2 des Statuts von Extremadura). ${ }^{64}$ Die Entscheidung des Verfassungstribunals zum katalanischen Statut 2010 betrachtete diese Referendumsbestimmungen in einem obiter dictum als verfassungskonform. Es bestätigte damit, dass sie als Teil der Kompetenz zur Regelung des Verfahrens von Statutsänderungen gemäß Art. 147 Abs. 3 spanVerf ganz eindeutig in den Bereich der regionalen Autonomie fallen. ${ }^{65}$

\section{Schlussfolgerungen}

$[D]$ emocracy is an endless journey, and, therefore, it may be more appropriate to speak, not so much about total democracy, but about the democratic route, that is, a progressive evolution of political systems to meet citizens' demands for participation. ${ }^{66}$

Im Sinne dieses dynamischen Verständnisses des Zusammenhanges zwischen Demokratie und Partizipation führte die "Route der Demokratie in Spanien seit 1978 ganz eindeutig in die Richtung von mehr direkter Bürgerbeteiligung in verschiedensten Formen. Dieser allgemeine Befund wird häufig - zumindest teilweise - auf einen Trend zur Politikverdrossenheit zurückgeführt, der als desafección politica Eingang in das gängige politische Vokabular gefunden hat, aber natürlich kein auf ein einzelnes Land beschränktes Phänomen darstellt. ${ }^{67}$ Unter anderem wird diese Unzufriedenheit auch darauf zurückgeführt, dass das seit 1978 herrschende vorwiegend repräsentative Demokratiemodell im Sinne des obigen Zitats die veränderten Ansprüche der Bürger an die erstrebenswerten Formen politischer Partizipation nicht mehr gerecht wird und dieses Modell daher einer Ergänzung bedarf.68

Diese Suche nach einer, wenn nicht idealen, so doch besseren Mischung zwischen repräsentativer, direkter und partizipativer Demokratie und auf einer tieferliegenden Ebene - nach der Identität von Demokratie im

64 Serra und Oñate, »The Reform of the Spanish Subnational Constitutions« (Fn. 4).

65 STC 31/2010, FJ 147.

66 Daniele Archibugi, »Principles of Cosmopolitan Democracy«, in Daniele Archibugi, David Held und Martin Köhler (Hrsg.), Re-Imagining Political Community (Polity Press, 2000), 200.

67 Vgl. Gerry Stoker, »The Rise of Political Disenchantment«, in Colin Hay (Hrsg.), New Directions in Political Science: Responding to the Challenges of an Interdependent World (Palgrave Macmillan, 2010), $43 \mathrm{ff}$.

68 Vgl. Rafael Rubio Núñez, »La guerra de las democracias«, (2007) 16 Asamblea: Revista Parlamentaria de la Asamblea de Madrid 79, 79ff. 
21. Jahrhundert kommt global insbesondere auch in den unzähligen »Bindestrich-Demokratiebegriffen" der letzten Jahre zum Ausdruck. Begriffe wie "post-democracy«, »audience-democracy oder »unpolitical democracy« sind nur einige Beispiele unter vielen. ${ }^{69}$ Die nicht nur auf Spanien beschränkte Identitätskrise der Demokratie ist vielmehr in großem Ausmaß gewissen strukturellen Veränderungen geschuldet. Eine Bürgerschaft, die über die letzten Jahrzehnte von einem tendenziell breiteren Zugang zu Bildung und Information profitierte, ist durch »kognitive Mobilisierung « ${ }^{70}$ immer weniger gewillt, politische Entscheidungen ausschließlich gewählten Repräsentanten zu überlassen. Ein weiterer bedeutender struktureller Faktor ist die steigende Komplexität von Entscheidungen, zurückgeführt auf eine »explosion of fact «. ${ }^{71}$ Diese Komplexität ist von gewählten Vertretern allein schwer zu bewältigen und erfordert die Einbeziehung von Bürgern in demokratische Prozesse, aber auch von kollektiven privaten Akteuren wie Interessensvertretungen, Expertengruppe und NGOs.

In Bezug auf beide Formen der Ergänzung der repräsentativen Demokratie, d.h. direkte und partizipative Demokratie, kann ein föderales System insofern von Vorteil sein, als es die kleinräumige Anwendung ihrer Instrumente auf einer regionalen Ebene ermöglicht. ${ }^{72}$ Dieses in anderen Ländern sehr wohl ausgeschöpfte Potenzial wird in Spanien jedoch nur in ziemlich geringem Ausmaß realisiert. Wie dieser Beitrag zeigt, wird die seit 1978 auf der nationalen Ebene etablierte Vorherrschaft der repräsentativen Demokratie auf der regionalen Ebene kaum gebrochen. Die Tatsache, dass die AG zwar Ansätze eigener Demokratiemodelle aufweisen, aber nicht viel mehr, scheint verschiedenen Faktoren geschuldet zu sein.

Erstens bilden die spanische Verfassung und die auf ihrer Basis getroffenen organgesetzlichen Regelungen teilweise ein sehr enges rechtliches Korsett. Die besten Beispiele hierfür sind wohl Art. 87 Abs. 3 spanVerf zur Volksinitiative i.V.m. den Organgesetzen von 1984 und 2006 sowie Art. 149 Abs. 1 Z. 32 spanVerf zur staatlichen Zuständigkeit für die Genehmigung der Einberufung einer Volksbefragung auf dem Wege des Referen-

$69 \mathrm{Zu}$ diesen Begriffen vgl. Colin Crouch, Post-Democracy (Polity Press, 2004), 6; Bernard Manin, The Principles of Representative Government (Cambridge University Press, 1997), 223; Nadia Urbinati, Democracy Disfigured: Opinion, Truth, and the People (Harvard University Press, 2014), 81.

70 Ronald Inglehart, The Silent Revolution: Changing Values and Political Styles among Western Publics (Princeton University Press, 1977), $295 \mathrm{ff}$.

71 Clifford Geertz, Local Knowledge: Further Essays in Interpretive Anthropology (Basic Books, 2000), 171.

72 Vgl. Palermo und Kössler, Comparative Federalism (Fn. 7), 117ff. 
dums. Gerade am Fall der verschiedensten Formen von Volksbefragungen lässt sich aber ein zweiter limitierender Faktor erkennen. Schließlich zieht die ständige Rechtsprechung des Verfassungstribunals den AG enge Grenzen, indem sie auch hinsichtlich rechtlich nicht verbindlicher Abstimmungen sowohl die Genehmigung als auch die Regelung als nationale Zuständigkeit betrachtet. ${ }^{73}$ Eine weitere auffallend restriktive Auslegung betrifft das in Art. 23 Abs. 1 spanVerf verankerte Recht auf Partizipation, zumal die taxative Aufzählung der unter diese Bestimmung fallenden Instrumente politischen Teilhabe insbesondere die in den seit 2006 reformierten Autonomiestatuten eingeführte administrative Partizipation nicht umfasst. ${ }^{74}$ Ein dritter Grund dafür, dass es lediglich Ansätze eigener regionaler Demokratiemodelle gibt, liegt allerdings jenseits des zentralstaatlichen Einflusses durch verfassungs- und organgesetzliche Vorgaben bzw. die Judikatur des Verfassungstribunals, nämlich bei den AG selbst. Die teilweise mangelnde Nutzung ihres autonomen Gestaltungsspielraums zeigt sich insbesondere am Beispiel der Volksinitiative, bei der die Regelungen der Regionen - in mehr oder weniger großem Ausmaß, aber doch weitgehend - jener auf der nationalen Ebene entspreche. Dass Demokratie in Spanien seit 1978, wie vielfach beanstandet, von einer gewissen Fortschrittsfeindlichkeit (»inmovilismo «) ${ }^{75}$ geprägt ist und nach wie vor Parlaments- und Parteienherrschaft impliziert, ist also letztendlich nicht nur auf die Zentralregierung sondern auch auf die AG zurückzuführen.

73 STC 103/2008, STC 31/2010.

74 STC 119/1995, STC 247/2007, STC 31/2010.

75 Reyes Pérez Alberdi, »Democracia representativa«(Fn. 40), 2. 
